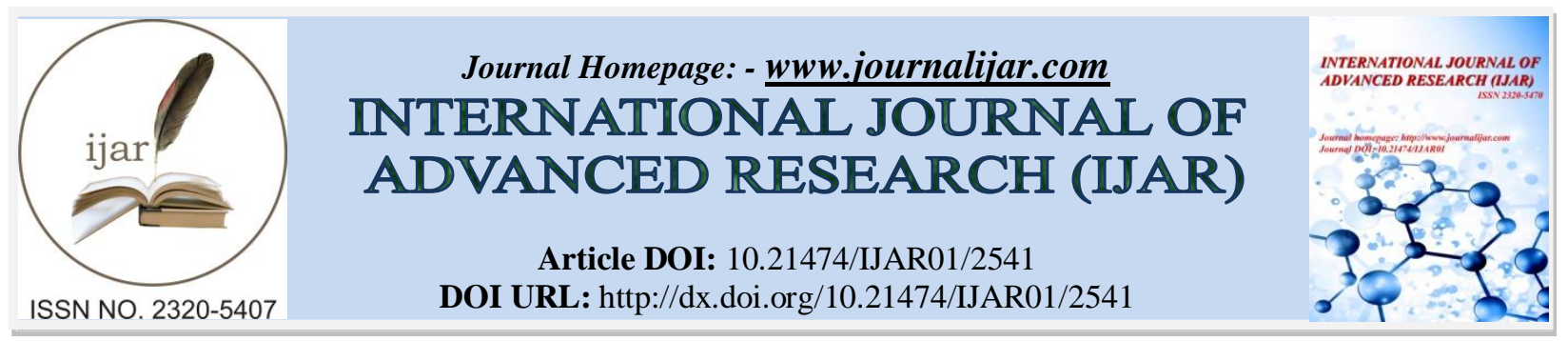

RESEARCH ARTICLE

\title{
DIGITAL BREAST TOMOSYNTHESIS AND DIGITAL MAMMOGRAPHY FOR BREAST CANCER DIAGNOSIS
}

Manar Moustafa Nasr, Wael Hamza kamr and Amal Abdelsattar Sakrana.

\section{Manuscript Info}

Manuscript History

Received: 25 October 2016

Final Accepted: 23 November 2016

Published: December 2016

Key words:-

Tomosynthesis, digital mammography,

BIRADS Classification, breast cancer.

\section{Abstract}

Purpose:- To compare the diagnostic performance of digital breast tomosynthesis (DBT) and digital mammography (DM) for breast cancer. Materials and Methods: Ninety patients from December 2013 till December 2015 were enrolled in the study. All patients had pathologically proven cancer. Assessment of the lesions based upon digital tomosynthesis, compared with digital mammography (DM) and correlated with the pathological results. Results: BT shows better detection of malignant speculated masses $(75 \%$ on DCI, $76.47 \%$ on ILC, $84.38 \%$ on IDC compared with $(12.5 \%, 41.18 \%, 67.19 \%$ on DM respectively). Fourteen malignant masses were clearly visible on BT while were questionably visible on DM. BT changes BIRADS classification from IV to $\mathrm{V}$ in 22 patients $(24.44 \%$ more accurate; 81.11/56.66\%)).

Conclusion: Tomosynthesis improves the detection and characterization of breast masses, especially in dense breast.

Copy Right, IJAR, 2016,. All rights reserved.

\section{Introduction:-}

Mammography is the most standard technique in the diagnostic imaging of the breast (1).

The sensitivity of conventional 2D mammography has reported to be $80-90 \%$ and decreased to about $48 \%$ in extremely dense breasts (2). It is a method that breast tissue is visualized as a two dimensional image (3). Parenchymal overlap which may cause significant obstacle to detect abnormal lesion is often met in conventional mammography (4).

In 3D digital breast tomosynthesis (BT), tomographic images are reconstructed from multiple projections taken from different angles (5). This technique allows the generation of 3D data and resolves the problem of tissue overlap (6).

BT improve the diagnostic accuracy by better visualization of the low contrast features that are hard to depict in conventional mammography due to breast tissue overlap $(7,8)$.

The main aim of our present study was to compare the diagnostic performance of breast tomosynthesis with conventional mammography. 


\section{Materials and Methods:- \\ Patientpopulation:-}

Patients were selected from among two groups during the period of December 2013 to December 2015:

1. Patients came for diagnostic digital mammography for assessment of a lump or other symptoms (78 patients).

2. Patients of high risk group came for screening mammogram (12patients).

\section{Methods:-}

Imaging modalities:-

Combined conventional mammography and tomosynthesis as well as ultrasonography were done for all patients.

All breast cancers diagnoses were verified by US guided core biopsy.

\section{Imageacquisition:-}

Mammography:-

BilateralDM and tomosynthesis were performed on a dedicated mammography unit; Mammomat inspiration Siemens', Erlangen, Germany.

Combo 2D and 3D technique with craniocaudal and mediolateral oblique views of both breasts was performed. On each view, traditional 2D image was taken. On tomosynthesis, average 25 low dose images (20-30) are acquired at different projection from which average of sixty four images (52-76) were performed, each image is about $1 \mathrm{~mm}$ thickness. They are taken from projections over an angular range of 180 degrees.

The mean absorbed dose for DM of a standard breast was $0.9 \mathrm{mGy}$.

The detector was operated in full resolution mode, thus the data acquisition time was of 20 s per breast. Image reconstruction was performed by filtered back-projection.

\section{Ultrasonography:-}

Bilateral whole breast $\mathrm{M}$ mode ultrasonography was performed with the guidance of clinical and mammographic findings. Linear array broadband transducer with frequency of $10 \mathrm{MHz}$ was used.

Survey scanning was performed in radial, transverse and sagittal planes.

US examinations were performed on a dedicated unit (Philips IU $22 \mathrm{X}$ matrix).

US guided core biopsy was performed for all patients using the same dedicated machine of US study. The core needle biopsy was done using a biopsy gun with $16 \mathrm{~g}$ needle in 56 patients and $18 \mathrm{~g}$ needle in 34 Patients.

\section{Viewing conditions and interpretation:-}

$>$ Combined 2D and tomosynthesis were reviewed on an investigational prototype version of a DM Mammo report workstation, Siemens', Erlangen, Germany.

$>\mathrm{DM}$ and tomosynthesis were analyzed separately. On each, breast lesions were analyzed according to the presence of asymmetry, mass, architectural distortion, calcification, or combinations of these signs. Then, the findings were classified according to the Breast Imaging Reporting and Data System (BIRADS lexicon) once on the basis of DM images and then on tomosynthesis and the causes of classification were listed.

$>$ The criteria of the suspected masses were reviewed on US according to the shape, outline, echogenecity, posterior shadowing and the surrounding parenchyma. Each item is evaluated and registered.

$>$ US findings were correlated to the routine mammographic findings and modify BIRADS classification in 23 patients.

$>$ Comparison was performed between tomosynthesis and DM as well as between combined tomosynthesis and 2DM with US findings.

$>$ Ultrasonography was reviewed on two 6- mega-pixel flat-panel BARC monitors. They were calibrated to the DICOM Grayscale Standard Display function. DM, tomosynthesis and US images were available on the workstation.

Dastly, tomosynthesis and overall imaging opinions were compared with the pathological results. 


\section{Results:-}

Our study included 90 women with average age 50.5 years (range from 29 to 72 years old). Twelve women (about $13 \%$ ) were selected from screening and the remaining patients (78 about 87\%) were selected from patients came for diagnostic studies.

From the second group, 48 patients had palpable masses (about 61.5\%), 19 patients had breast pain (24.4\%), 7 patients had nipple secretions (8.97\%) and 4 patients had skin or nipple changes $(5.1 \%)$.

\section{Detection of the lesion by DM and DBT with comparison to the pathological results:-}

Out of all 90 breast cancers, 64 (71.1\%) were invasive ductal carcinoma (IDC), 17 (18.8\%) invasive lobular carcinoma (ILC), 8 (8.89\%) ductal carcinoma in situ (DCIS), and 1 (1.11\%) intracystic papillary carcinoma (ICPC). The average size of the detected masses was $42 \mathrm{~mm}$ (range 5-80 $\mathrm{mm}$ ).

Table 1:- Ninety malignant breast lesions by appearance on DM and DBT.

\begin{tabular}{|l|l|l|}
\hline Radiological appearance & DM & DBT \\
\hline Focal asymmetry & $2(2.22 \%)$ & - \\
\hline Architectural distortion & $6(6.67 \%)$ & $2(2.22 \%)$ \\
\hline Dense mass & $51(56.67 \%)$ & $57(63.33 \%)$ \\
\hline Microcalcifications only & $2(2.22 \%)$ & - \\
\hline Mass and calcifications & $29(32.22 \%)$ & $31(34.44 \%)$ \\
\hline
\end{tabular}

In Table 1 the appearance of the breast lesions on DM and 3DBT are listed. Two cases revealed focal asymmetrical area of increased density on DM were seen as spiculated masses on DBT (Fig 1), they are proved pathologically to be ILC. DBT revealed masses in four cases with architectural distortion, the masses appeared dense and irregularly outlines (Fig 2), They were proved pathologically to be DCI (3 cases) and ILC (one patient). Out of the 51 dense masses detected by DM, thirteen masses were appears microlobulated with no clear speculation or infiltration, were clearly seen by DBT as partially speculated and infiltrated masses. Micro-calcifications were more clear at DBT than DM and associated masses were detected on two patients by DBT were not clear by DM. Both are proved pathologically to be IDC. Table 2 shows the pathological results and the corresponding radiological appearance.
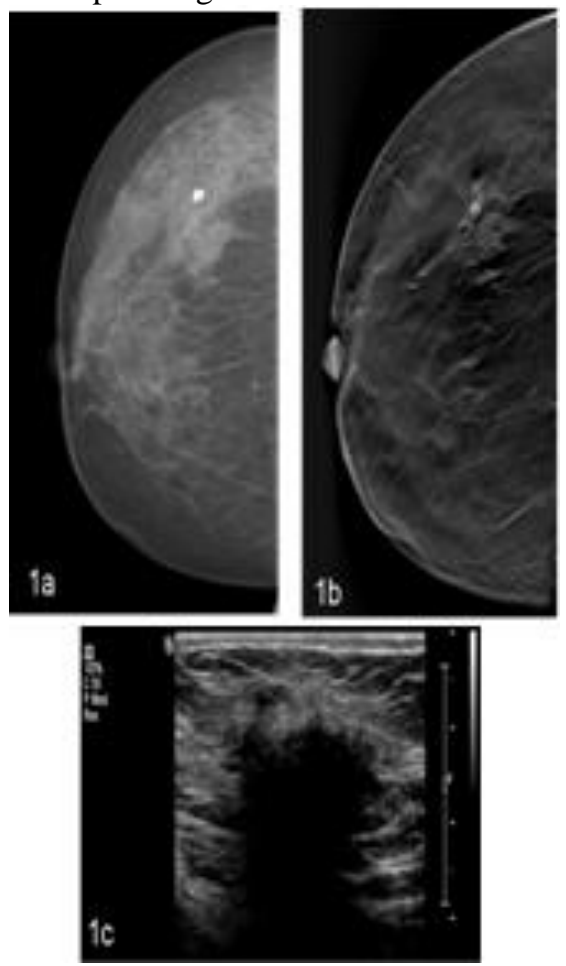

Fig 1:- (a) DM CC views shows a large dense area at the upper outer quadrant of the right breast with coarse calcifications. (b) BT shows better delineation of the margins of the speculated mass with surrounding parenchymal 
infiltration. (c) US shows a large hypechoeic mass with irregular speculated margin and posterior acoustic shadowing.

Table 2:- shows the pathological result of the lesions and the corresponding appearance by DM and DBT.

\begin{tabular}{|c|c|c|}
\hline Pathological result & DM & DBT \\
\hline DCI (8) $(8.89 \%)$ & $\begin{array}{l}3 \text { architectural distortion }(37.5 \%) \\
4 \text { non speculated masses }(50 \%) \\
1 \text { spiculated mass }(12.5 \%)\end{array}$ & $\begin{array}{l}1 \text { architectural distortion }(12.5 \%) \\
1 \text { non speculated mass }(12.5 \%) \\
6 \text { spiculated masses }(75 \%)\end{array}$ \\
\hline ILC (17) (18.89\%) & $\begin{array}{l}2 \text { focal asymmetry }(11.76 \%) \\
3 \text { architectural distortion }(17.65 \%) \\
5 \text { non speculated mass }(29.41 \%) \\
7 \text { spiculated mass }(3+\mathrm{Ca})(41.18 \%)\end{array}$ & $\begin{array}{l}1 \text { architectural distortion }(5.88 \%) \\
2 \text { non speculated mass }(11.76 \%) \\
13 \text { spiculated mass }(3+\mathrm{Ca})(76.47 \%)\end{array}$ \\
\hline IDC (64) (71.11\%) & $\begin{array}{l}2 \text { Ca only }(3.16 \%) \\
17 \text { non speculated mass }(26.56 \%) \\
43 \text { spiculated mass }(26+\mathrm{Ca})(67.19 \%)\end{array}$ & $\begin{array}{l}10 \text { non speculated mass }(15.63 \%) \\
54 \text { spiculated mass }(28+\mathrm{Ca})(84.38 \%)\end{array}$ \\
\hline ICPC (1) (1.11\%) & Non speculated mass & Non speculated mass \\
\hline
\end{tabular}

\section{Cancer visibility:-}

The malignant mass visibility was analyzed on both DBT and DM and shows that Tomosynthesis detected the presence of dense mass which was not visible in the routine diagnostic DM 2 views in 8 patients (table 3 )
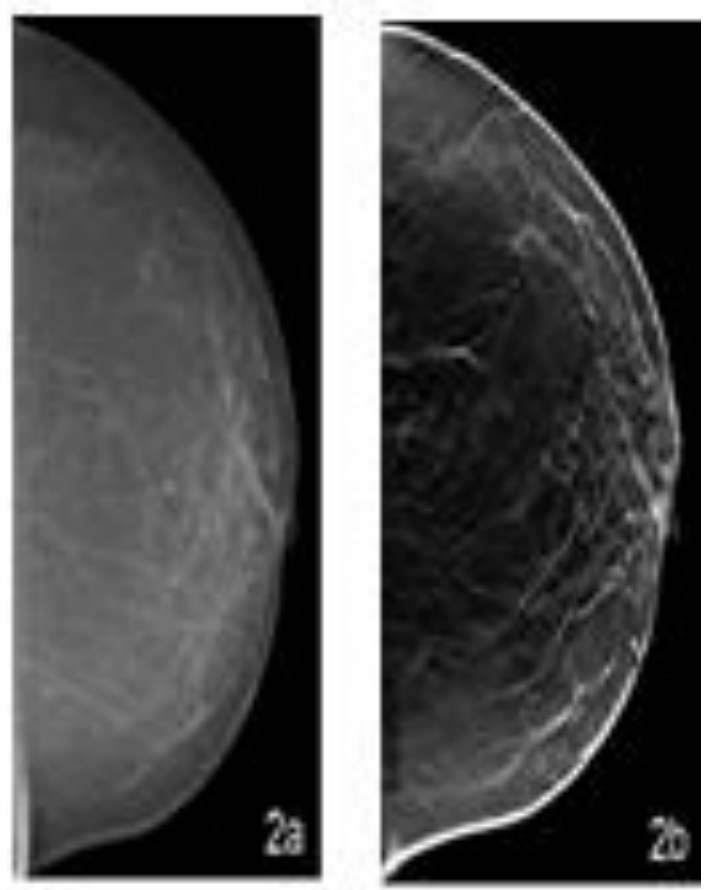

Fig. 2 A pathologically proven IDC appears as a small fairly dense area of architectural distortion at the upper outer quadrant of the left breast on DA (a). (b) BT CC view shows a speculated mass with obvious infiltration to the surrounding parenchyma. 
Table 3:- shows the visibility of the ninety malignant masses on both DBT and DM.

\begin{tabular}{|l|l|l|}
\hline DM & DBT & No of masses \\
\hline Not visible & Questionably visible & 2 \\
\hline Not visible & Clearly visible & \multicolumn{2}{|c|}{6} \\
\hline Questionably visible & Clearly visible & 14 \\
\hline Clearly visible & Clearly visible & 68 \\
\hline Clearly visible & Not or questionably visible & - \\
\hline
\end{tabular}

In 22 patients, the DBT increased the BIRADS classification from BIRADS IV (detected by DT with US) to V by detecting the mass infiltration and speculation more clear. (table 4)

So cancer visibility was higher by BT then the use DM $(91.11 \%$ / 75.55\%).
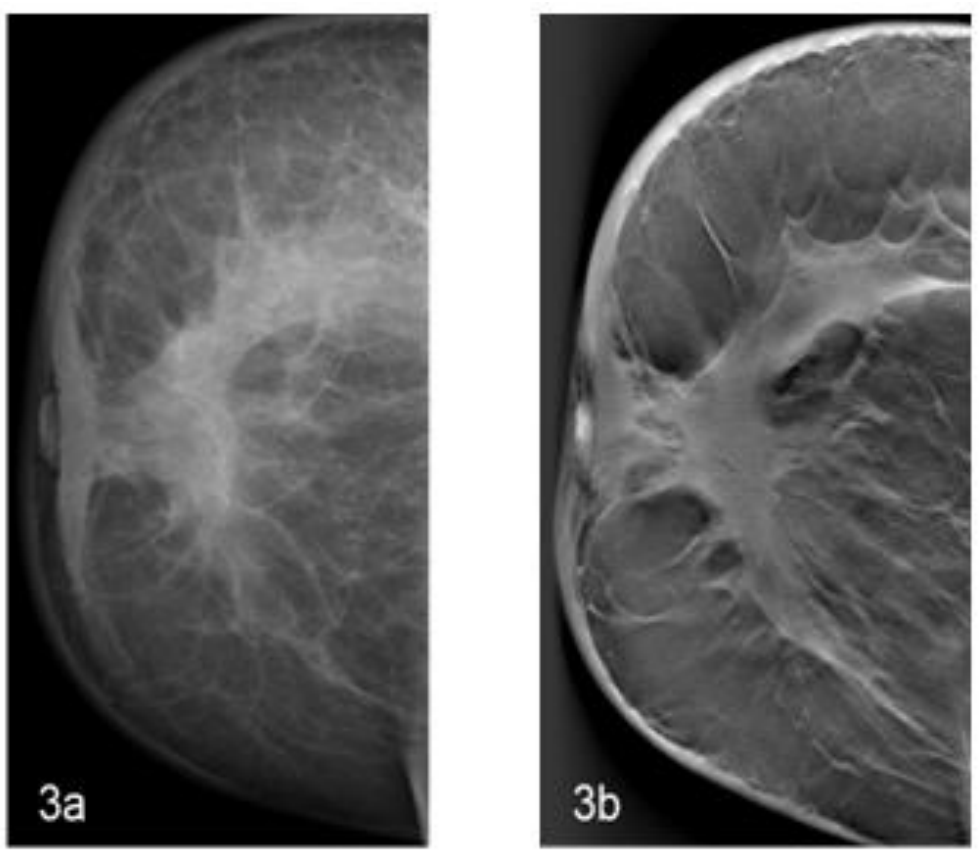

Fig. 3 A pathologically proven $I L C$ appears as increased density of the retroareolar region of the right breast on DM (a), associated with skin edema and nipple retraction. (b) BT CC view shows the mass speculation and infiltration more clear with more evident nipple retraction ; changing it from BIRADS IV by DM to V on BT.

Table 4:- shows the BIRADS classification of the 90 masses by both DBT and DM.

\begin{tabular}{|l|l|l|}
\hline BIRADS Classification & DM & DBT \\
\hline III & 1 & 1 \\
\hline IV & 38 & 16 \\
\hline V & $51(56.66 \%)$ & $73(81.11 \%)$ \\
\hline
\end{tabular}

BIRADS classification for breast lesions on DM and BT:-

DBT improve the visualization of mass infiltration to the surrounding parenchyma and therefore increased the BIRADS classification (Fig 3,4). In 38 patients classified as (BIRADS IV) by DM, 12 patients were ranked higher classification (BIRADS V) by BT.

The US done shows that the mass classified as DIRADS III in both digital mammography and tomosythesis is well defined capsulated large with intratumoral cysts and papillary projections. The pathological study revealed intracystic papillary carcinoma (ICPC).

Tomosynthesis was conclusive in about $73(81.11 \%)$ patients. 
The main purpose of this study was to compare the appearance of malignant breast lesions on DM and BT. The masses were better identified on BT and subsequently classified more accurately according to BIRADS classification.

The sensitivity of mammography is limited in dense breasts so lesions may be concealed by a moderate amount of dense parenchyma (9).
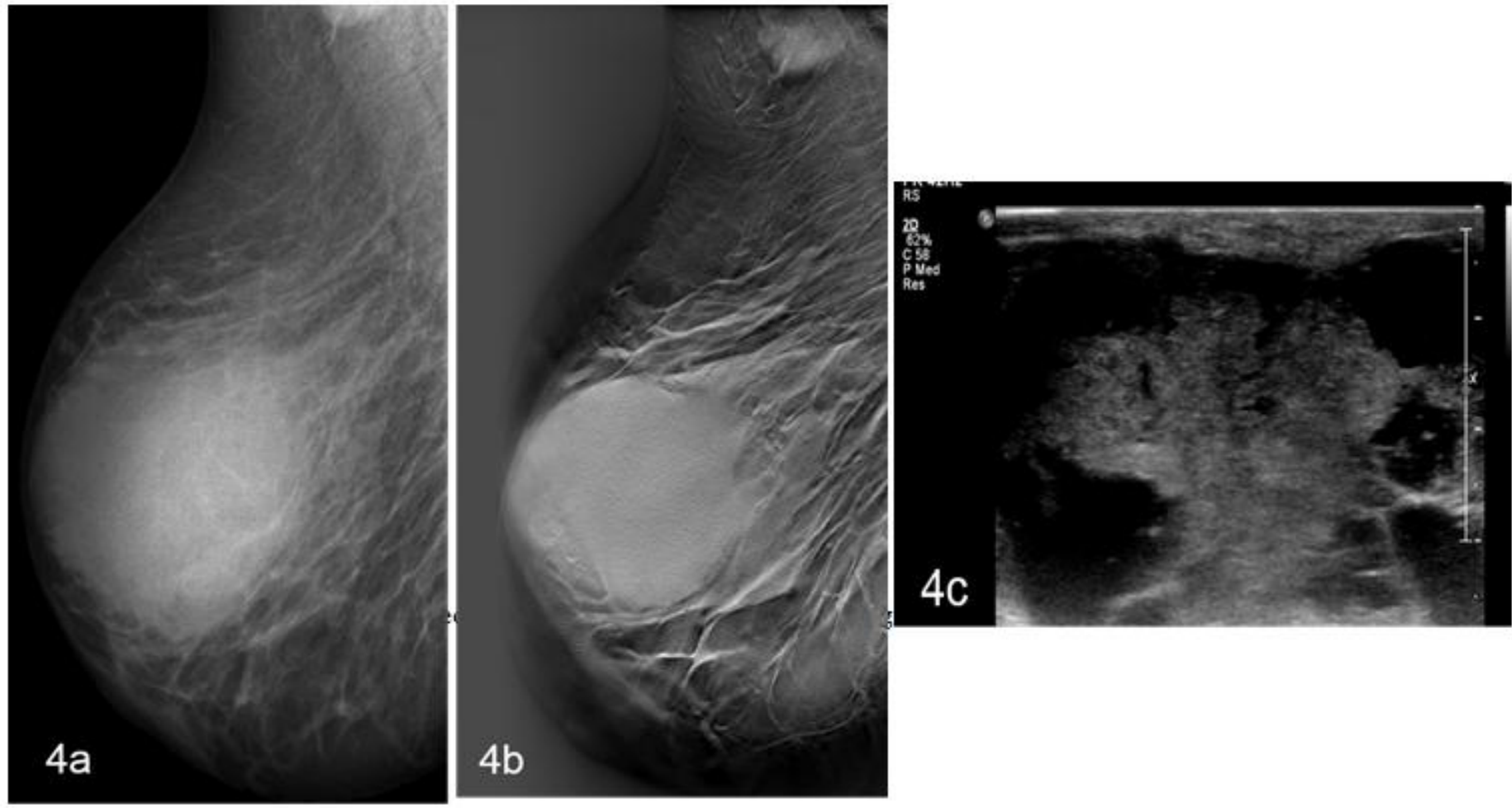

Fig 4:- (a) MLO view DM of the right breast shows a large rounded dense mass in the upper outer quadrant. By BT (b) ill definition of the posterior border of the mass was noticed with infiltration to the deep parenchyma (BIRAD V). (c) US shows a large cystic mass with intracystic large papillary projection forming a cauliflower appearance of the solid component. Core biopsy was proved to ICPC.

\section{Discussion:-}

Through our study, we compared the appearance of the mass including the detection and extension on DM and tomosynthesis.

Better delineations of the lesion border result in more accurate interpretation (10). Breast tomosynthesis overcomes the limitation of DM. The superimposition of fibroglandular tissues which hampers the detection of the lesion or limits the visualization of its criteria is reduced in tomographic technique (11).

It was reported that architectural distortion accounted for $12 \%$ to $45 \%$ of overlooked breast cancer in screening mammography (12). DBT was proved to be a method to solve this problem and therefore lessen the false negative cases (13).

The problem of obscuring superimposed tissue may be more complicated by the density and morphology of the mass (14). We noticed that the areas of architectural distortion (Fig 3) as well as large rounded masses (Fig 4) were more affected by the tissue overlap at DM than the speculated masses. The malignant mass embedded in the dense tissue was the main limiting factor explaining the non visibility of the lesion.

At DCIS with invasive IDC, the invasive portion is usually not possible to identify by DM due to attenuation differences (14). 
We noticed malignant masses characterized by pleomorphic calcifications; the non calcified large invasive component was difficult to identify due to lack of edge characteristics, because it is usually has a non specific growth pattern and obscured by the surrounding tissue (Fig 5).
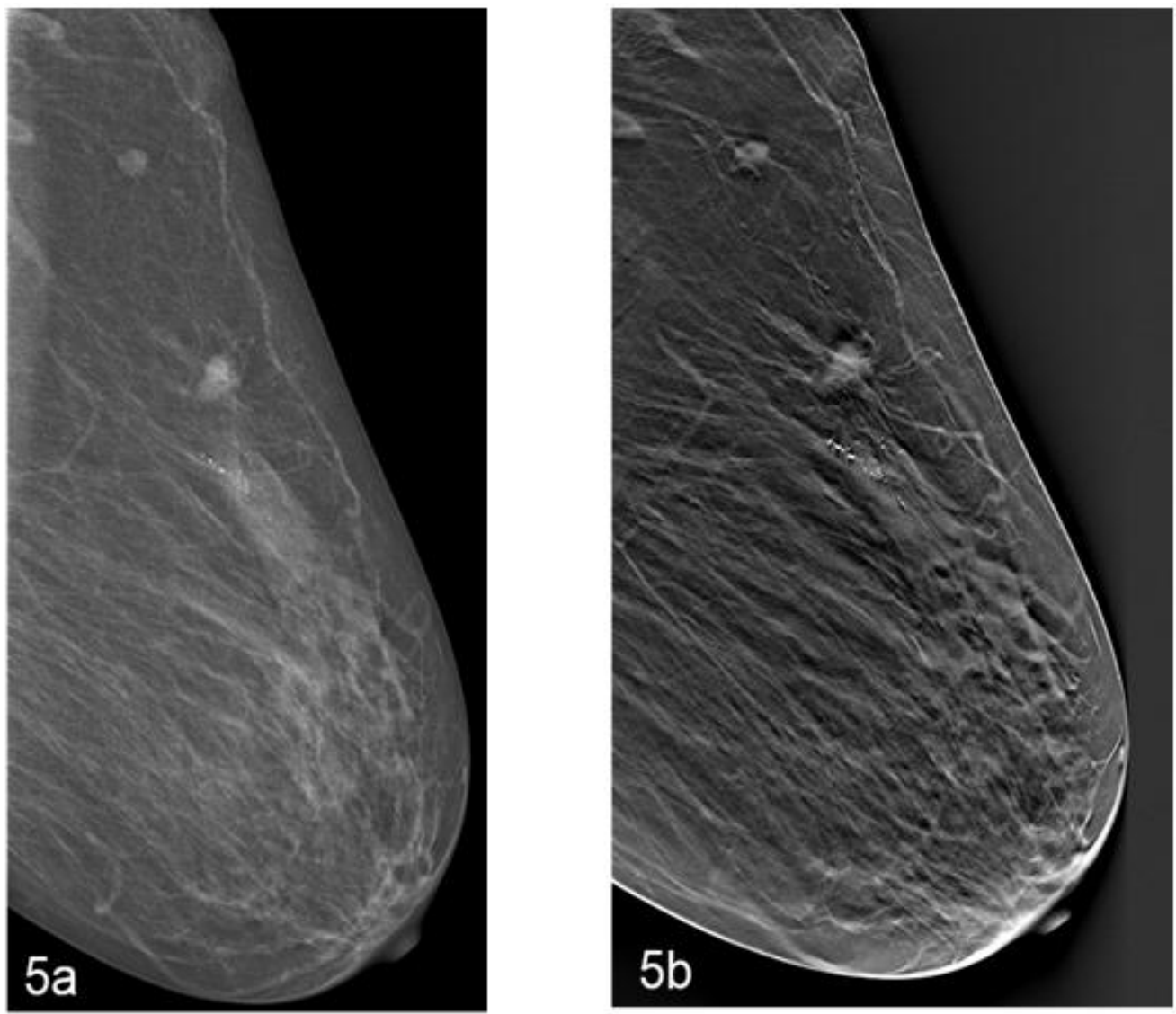

Fig. 5:- (a) DM MLO view of the left breast shows a dense area of architectural distortion with cluster of microcalcifiation is noted at the upper outer quadrant. A dense lobulated mass seen above this lesion. By BT (b) MLO view shows better visualization of the dense mass which shows band of surrounding parenchymal infiltration from its margins. Also the calcification and the related dense area is more demarcated (BIRAD V).

BT was conclusive in 73 patients $(81.11 \%)$ and the use of other modalities; US, elastography or MRI didn't give us more information or not needed for more accurate BIRADS classification.

\section{Conclusion:-}

From our study, the results indicates that BT has higher sensitivity for breast cancer detection than two views DM. It also helps in more accurate BIRADS classification of the mass even in dense breasts, overcoming the major problem of tissue superimposition at DM. 


\section{References:-}

1. Humpherey LL, Helfand M, Chan BK and Woolf SH:Breast cancer screening. A summary of the evidence for the US. Preventive services task force. AnnInternMed. 2002;137, 347-60.

2. Birdwell RL, Ikeda DM, O'Shaughnessy KF and Sickles EA: Mammographic characteristics of 115 missed cancers later detected with screening mammography and the potential utility of computer aided detection. Radiology. 2001; 219: 192-202.

3. Kerlikowke K, Grady D, Barclay J, Sickles EA and Ernester V: Effect of age, breast density and family history on the sensitivity of screening mammography. JAMA. 1996; 276: 33-38.

4. Carney PA, Miglioretti DL, Yankaskas B,, Kerlikowske K, Rosenberg R, Rutter CM et al,: Individual and combined effects of age,breastdensity,andhormone replacement theraphy use on the laccuracy of screening mammography. Ann Intern Med. 2003; 138, 168-75.

5. Niklason LT, Christian BT, Niklason LE, Kopans DB, Castleberry DE, Opsahl-Ong BH et al, Digital tomosynthesis in breast imaging. Radiology. 1997; 205: 399-406.

6. Skaane P, Bandos AI, Gullien R, Eben EB, Ekseth U, Haakenaasen Uet al,:Comparison of digital mammography alone and digital mammography plus tomosynthesis in a population-based screening program. Radiology. 2013; 267, 47-56.

7. Michell MJ, Iqbal A, Wasan RK,Evans DR, Peacock C, Lawinski CPet al,: A comparison of the accuracy of film-screen mammography, full-field digital mammography, and digital breast tomosynthesis. ClinRadiol. 2012; 67, 976-81.

8. Rafferty EA: Breast tomosynthesis. Adv digital radiography: RSNA Categorical course in Diagnostic Radiology Physics. 2003; 291-226.

9. Kolb TM, Lichy J, Newhouse JH: Comparison of the performance of screening mammography, physical examination, and breast US and evaluation of factors that influence them: an analysis of 27,825 patient evaluations. Radiology. 2002; 225:165-175.

10. Zuley ML, Bandos AI, Ganott MA, Sumkin JH, Kelly AE, Catullo VJet al, :Digital breast tomosynthesis versus supplemental diagnostic mammographic views for evaluation of non calcified breast lesions. Radiology. 2013;266: 89-95.

11. Andersson I, Ikeda DM,Zackrisson S,RuschinM, Svahn T, Timberg Pet al,: Breast tomosynthesis and digital mammography; a comparison of breast cancer visibility and BIRADS classification in a population of cancers with subtle mammographic findings. Euro Radiol. 2008; 18:2817-2825.

12. Burrell HC, Evans AJ, WilsonAR, Pinder SE:False negative breast screening assessment. What lessons can we learn ? Clinical radiology. 2001; 56 No 5: 385-388.

13. Skaane P, Gullien R, Bjorndal H, Eben EB, Ekseth U, Haakenaasen Uet al,: Digital breast tomosynthesis (DBT): initial experience in a clinical setting. ActaRadiol. 2012; 53: 524-529.

14. Hakim CM, Chough DM, Ganott MA, Sumkin JH, Zuley ML, Gur D:Digital breast tomosynthesis in the diganostic environment: a subjective side by side review. AJR Am J Roentegenol. (2010); 189: 614-619.

\section{Conflict of Interest:-}

The authors declare that they have no conflict of interest. 